\title{
Protein Palmitoylation and Pathogenesis in Apicomplexan Parasites
}

\author{
Maria Martha Corvi, Andres Mariano Alonso, and Marina Cecilia Caballero \\ Laboratorio de Parasitologia Molecular, Instituto Tecnológico de Chascomús (IIBINTECH), \\ CONICET-Universidad Nacional de General San Martín, Intendente Marino Km 8,2, P.O. Box 164, \\ Chascomús, B7130IWA Buenos Aires, Argentina
}

Correspondence should be addressed to Maria Martha Corvi, mcorvi@intech.gov.ar

Received 23 May 2012; Revised 18 July 2012; Accepted 24 July 2012

Academic Editor: Andrea Silvana Rópolo

Copyright ( 2012 Maria Martha Corvi et al. This is an open access article distributed under the Creative Commons Attribution License, which permits unrestricted use, distribution, and reproduction in any medium, provided the original work is properly cited.

\begin{abstract}
Apicomplexan parasites comprise a broad variety of protozoan parasites, including Toxoplasma gondii, Plasmodium, Eimeria, and Cryptosporidium species. Being intracellular parasites, the success in establishing pathogenesis relies in their ability to infect a hostcell and replicate within it. Protein palmitoylation is known to affect many aspects of cell biology. Furthermore, palmitoylation has recently been shown to affect important processes in $T$. gondii such as replication, invasion, and gliding. Thus, this paper focuses on the importance of protein palmitoylation in the pathogenesis of apicomplexan parasites.
\end{abstract}

\section{Introduction}

It is well known that unicellular eukaryotes are the simplest models for the study of molecular mechanisms operating in the whole domain. Apicomplexan parasites are a large group of unicellular protozoan parasites, which include Toxoplasma gondii, Plasmodium, Eimeria, and Cryptosporidium spp. These parasites are the cause of great public health problems affecting both humans and animals [1]. These diseases have high morbidity and mortality rates [2-4] and affect about one third of the global population. Toxoplasma gondii alone is responsible for the infection of 2 billion people worldwide, and the symptoms of toxoplasmosis are determined by the immune status of the individual [5]. All members of the phylum are characterized by the presence of a unique organelle called apicoplast and an apical structure. Whereas the apicoplast is involved in lipid metabolism [6-8], the apical structure has a role in the invasion of the host-cell (reviewed in $[9,10]$ ).

Since most protozoa of the Apicomplexa phylum are obligate intracellular parasites, host-cell invasion, replication and egress are vital requirements for the establishment of pathogenesis. The ability of the tachyzoite stage to invade, replicate, and egress the host-cell repeatedly generating tissue damage and inflammation is the basis of human and animal symptoms. To date, the complex machinery involved in the invasion process has been more or less characterized for the different members of the phylum [11]. Besides, it is known that invasion of the host-cell is a highly coordinated process and most of the proteins that participate in it feature posttranslational modifications that regulate their localization and/or function. Another contributing factor to parasite dissemination is its capacity to replicate once inside the host-cell. In fact, in T. gondii an increase in the multiplication rate has been linked to the acute virulence in mice $[12,13]$. The replication process in $T$. gondii has been thoroughly reviewed elsewhere $[14,15]$.

Posttranslational modifications add diversity to the proteome. These dynamic modifications affect almost all aspects of cell biology and play an important role in protein transport, cellular localization, and regulation of enzymatic activity. Among these modifications we can find glycosylation, alkylation, methylation, acetylation, phosphorylation, ubiquitination, and protein lipidation. Although protein phosphorylation represents the vast majority of regulated covalent modifications observed [16], the covalent modification of proteins by lipids (protein lipidation) is 
an emergent topic. Protein lipidation alters the physical and functional properties of a protein, depending on the type of lipid attached to it. This functional diversity is provided by four categories of protein lipidation: prenylation, glypiation, cholesteroylation, and fatty acylation [17, 18]. With the exception of cholesteroylation, all other lipidations have been described to operate in apicomplexan parasites [19], and some of them are even targets for the treatment of malaria (recently reviewed in $[20,21]$ ). Protein fatty acylation refers to the covalent modification of proteins by fatty acids and is divided into two categories: myristoylation and palmitoylation [17]. Whereas myristoylation is the irreversible coor posttranslational attachment of myristic acid (C14:0) onto proteins via a stable amide bond [22, 23], protein palmitoylation is defined as the reversible posttranslational attachment of palmitate (C16:0) via a thioester linkage [24]. Protein myristoylation has been reported in many apicomplexan parasites such as Eimeria tenella [25] and Plasmodium falciparum [26, 27]. Although palmitoylation usually occurs on a cysteine residue, less frequently it has also been shown to occur on N-terminal amino acids via an amide bond $[28,29]$. Palmitoylation is the only reversible lipid modification and as such, it can regulate the fate of the targeted proteins. In the recent years, the study of posttranslational modifications and more precisely protein palmitoylation has gained importance in this group of protozoan parasites [30]. As such, in the following sections of this paper we will focus on the role of protein palmitoylation in different processes involved in the pathogenesis of apicomplexans.

\section{Protein Palmitoylation}

Our comprehension of protein palmitoylation and its function is based on studies in higher eukaryotes. A short review of the most important roles of this posttranslational modification is described below.

Palmitoylation is an important mechanism for regulating subcellular localization [31, 32], trafficking (recently reviewed in [33]), translocation to lipid rafts $[34,35]$, regulation of enzymatic activity $[36,37]$, and gene expression [38]. Before its attachment to the targeted protein, palmitate must be activated in its coenzyme A derivative, palmitoylCoA [39]. The reversible nature of this modification acts as a molecular switch that dynamically controls and regulates either the localization and/or function of selected proteins $[40,41]$. Besides, it is important to highlight that palmitoylation does not have a clear sequence requirement other than a cysteine residue.

Interestingly, palmitoylation is found both on peripheral and transmembrane-spanning proteins. Integral membrane proteins are palmitoylated in either their cytosolic domain or at the transmembrane/cytosolic interface. In this type of proteins, palmitoylation has been found to be affected by the proximity to the lipid-ordered phase of the membrane, the composition of the transmembrane domain, and the length of the cytoplasmic tail $[42,43]$. Peripheral membrane proteins that are palmitoylated are sometimes myristoylated or prenylated as well. Myristoylation and prenylation occur in the cytosol and confer transient membrane interaction [44]. This transient association allows proteins to interact with palmitoyl acyl-transferases (PATs), integral membrane enzymes responsible for protein palmitoylation. Once palmitoylated, these proteins stably associate with membranes $[24,44]$. Members of the Ras superfamily of GTPases are prenylated and some members are also palmitoylated (reviewed in [33]). When palmitoylation is found with myristoylation, the palmitoylated cysteine residue is usually proximal to the N-terminal myristoylated glycine [22, 45]. Distinct from what occurs in integral membrane proteins, the amino acids surrounding the palmitoylated cysteine are not important when myristoylation takes place [45]. Members of the Src family of protein tyrosine kinases and $\mathrm{G}_{\alpha}$ are typical examples of this dual modification $[46,47]$. When palmitoylation is the only lipid modification, the modified cysteine is usually located within the first 25 amino acids. In this case, these proteins may possibly use an intrinsic weak membrane affinity for transient membrane interaction before palmitoylation occurs [48, 49].

Regarding the mechanism underlying protein palmitoylation, it has been reported to occur either spontaneously or to be enzyme-mediated. Examples of spontaneous palmitoylation are the mitochondrial proteins glutamate dehydrogenase, carbamoyl phosphate synthetase 1, and methylmalonate semialdehyde dehydrogenase where palmitoylation regulates their activities $[36,37,50]$. Recently, protein palmitoylation of the mitochondrial protein 3hydroxy-3-methylglutaryl-CoA synthase has been shown to play a role in gene regulation [38]. However, protein palmitoylation is mainly enzyme-mediated either by DHHCcontaining proteins (Asp-His-His-Cys-containing proteins; palmitoyl acyl-transferases; PATs) or membrane bound-Oacyltransferase (MBOAT) proteins. Whereas intracellular palmitoylation occurs by PATs, secreted proteins are palmitoylated by members of the MBOAT (membrane bound$\mathrm{O}$ acyl-transferase) family $[51,52]$. Genes encoding DHHC proteins are present in organisms from yeast to humans $[53,54]$. However, all these proteins have in general, some shared characteristics such as having an even number of transmembrane domains and to be expressed on the Golgi, ER, and the PM $[54,55]$.

Depalmitoylation can occur in response to signals or spontaneously [56]. In fact, an agonist-induced increase in palmitate turnover has been observed for proteins such as $\mathrm{H}$ Ras [57], $\mathrm{G}_{\alpha \mathrm{s}}$ [58], endothelial nitric oxide synthase [59], and PSD-95 [49]. Up to date, two lysosomal and one cytosolic thioesterases are thought to mediate protein depalmitoylation. However, only the cytosolic acyl-protein thioesterase1 (APT1) has been reported to mediate depalmitoylation in vivo. APT1 is a $29 \mathrm{kDa}$ nonspecific thioesterase that can remove fatty acyl groups from lysophospholipids as well as a wide variety of proteins [60-62]. In a recent report, Yang and colleagues showed that APT1 is palmitoylated, which may tether this protein to the membrane and thus, facilitate the depalmitoylation of its substrates [63]. The two lysosomal hydrolases palmitoyl-protein thioesterase I (PPT1) and PPT2 do not remove palmitate groups from palmitoylated proteins $[64,65]$ and as such, their substrates are unknown. 
Among the functions of palmitoylation are the attachment to membranes of otherwise soluble proteins and the traffic of lipid-modified proteins between different compartments. This sorting has been observed in the partitioning of proteins into lipid rafts $[34,35,66]$, ER-Golgi anterograde traffic (reviewed in [67]), flagella [68,69], and cilia [70, 71]. Another function of protein palmitoylation is the regulation of protein activity. Palmitoylation has been shown to regulate G-protein-mediated signaling at multiple levels: it regulates both the localization and the transducing signal of heterotrimeric $\mathrm{G}$ proteins (reviewed in [72]). Interestingly, the enzymatic activity of $\mathrm{N}$ - and $\mathrm{H}$-Ras proteins can be blocked in vitro by using inhibitors, for example, small molecules, targeting either a PAT type [73-75] or thioesterase [76]. As such, these enzymes are arising as interesting new targets for anticancer compounds. Mammalian and parasite PATs are homologous in the cysteine rich-domain that includes the DHHC motif but their $\mathrm{N}$-and C-terminal ends, which are thought to be responsible for either substrate specificity or interaction with a possible regulator, are completely different. Thus, it will be interesting to see the future development of PATs inhibitors capable of selectively blocking the apicomplexan counterparts without affecting the human host.

\section{Invasion in Apicomplexa}

The eukaryotic phylum Apicomplexa comprises more than 5000 species of parasitic protozoa, which have a wide range of vertebrate hosts. These pathogens have complex life cycles with several developmental stages. Most protozoa of this phylum are obligate intracellular parasites, for which hostcell invasion is a vital requirement. Even though apicomplexan parasites differ considerably in the type of host-cells they can infect, which include erythrocytes, lymphocytes, macrophages and even cells of the digestive tract from different animal species, they share conserved mechanisms of invasion and motility. Many aspects of invasion in Apicomplexa have thoroughly been reviewed elsewhere [9, $10,77]$. With the exception of Theileria, whose sporozoite and merozoite stages are not motile [78], most members of the phylum share a substrate-dependent locomotion called gliding. This locomotion requires the coordinated action of a multiprotein complex termed glideosome [79]. The glideosome is composed of adhesive proteins located between the parasite's plasma membrane (PM) and a pair of membranes known as the inner membrane complex (IMC). This type of motility is fundamental for cell invasion and tissue colonization $[9,80,81]$.

For invasion to take place, most apicomplexan parasites must release the content of their specialized apical secretory organelles (micronemes and rhoptries) [82]. After binding to the host-cell, the parasite forms a ring-like structure termed moving junction (MJ) between its apical tip and the host-cell membrane [9]. The MJ is the result of the interactions between mainly two well-conserved apicomplexan proteins: AMA1 (apical membrane antigen 1) and RON2 (rhoptry neck protein 2) [11]. Subsequent penetration and migration of the $\mathrm{MJ}$ is characterized by the formation of a parasitophorous vacuole (PV) around the parasite [83] that accommodates the invading zoite within the host cell. This specialized vacuole, however, is not always conserved throughout the intracellular parasite stage; the exceptions being Babesia bovis [84] and Theileria spp [78], which escape the PV once infection of the host-cell has been established. The fate of the parasite in the host cell is, therefore largely dependent on its ability to actively invade a cell by an MJdependent process.

\section{Protein Palmitoylation, Invasion, and Gliding in Apicomplexa}

The potential of apicomplexan parasites to colonize cells or tissues depends directly on their capability to move, adhere to, and invade the host cell in order to replicate within it and thus, ensuring the survival of the pathogen. Throughout the last decades, much effort has been dedicated to elucidate the intricate mechanisms underlying motility and invasion of these parasites. The increasing expansion of the knowledge of these peculiar eukaryotes allowed us to get a better understanding of their biology, yet new questions continuously arise. Ahead lies the study of the architecture of some these protein complexes, how they assemble, how each of their components get to their site of action, and how they act in such concerted way. One possibility that might shed light on these inquiries is the study of posttranslational modifications, in particular protein palmitoylation.

The first report of protein palmitoylation in Apicomplexa was performed on E. tenella where the protein cGMPdependent kinase (PKG) was shown to be palmitoylated [25] and to be the target of the broad-spectrum coccidiostat 4-[2-(4-fluorophenyl)-5-(1-methylpiperidine-4-yl)-1Hpyrrol-3-yl] pyridine. Interestingly, this compound was effective against coccidiosis in poultry and toxoplasmosis in a murine model $[85,86]$. Later studies on this modification came from the model organisms Plasmodium and Toxoplasma $[27,30,87,88]$. Recently, our group reported an in silico analysis performed on $T$. gondii proteins suggesting that many proteins involved in gliding and invasion may be palmitoylated and proposing the existence of at least 17 possible PATs [30]. Experimental support was further provided by a subsequent work that highlights the functional importance of protein palmitoylation on three processes that are crucial for the parasite's life cycle and, therefore, for its pathogenesis: gliding, invasion, and replication [87]. It was observed that 2-bromopalmitate-a nonselective lipid electrophile with selectivity towards reactive thiols widely used to study protein palmitoylation $[89,90]$ —decreased the invasive capacity of drug-treated tachyzoites. Even though the invasion process is somehow altered, the initial attachment to the host cell is not affected. Our in silico analysis allowed us to predict that two vital components of the MJ might be palmitoylated: AMA1 and RON2. Moreover, according to this study another micronemal protein with roles in invasion and virulence, MIC2 [91], may also be palmitoylated. Consequently, it is reasonable to infer that protein palmitoylation could play a relevant role in MJ 
assembly or in targeting its protein components to the cell membrane, enabling proper function of such relevant apparatus for invasion. Since 2-bromopalmitate exerts pleiotropic effects on cellular metabolism, a thorough study on the role of palmitoylation on these putative targets should be performed.

Recently, antigen mapping made on another member of the phylum, Eimeria maxima, identified a novel antigen: IMP-1 (immuno mapped protein-1). This protein shares features with AMA1 and, as such, can potentially induce the same immunoprotection [92]. Further in silico analysis showed the absence of a signal peptide or recognizable domain in IMP-1. Additionally, the homologous counterparts in T. gondii (XM_002370108) and Neospora caninum (NCLIV_000430) were found [92]. The latter was recently characterized by Cui and colleagues [93], who reported that palmitoylation and myristoylation operate on the protein, targeting it to the parasite's pellicle. Furthermore, the study stated that antibodies against NcIMP-1 inhibit parasite invasion, suggesting a role of this protein in invasion. The same group isolated the protein from $T$. gondii and localized it to the pellicle of the tachyzoite [94]. In addition, they propose TgIMP-1 as a possible vaccine candidate since the recombinant version of the protein stimulates both humoral and cell-mediated immunity [93].

As mentioned above, invasion is associated to gliding motility and, therefore, dependent on its glideosome. The protein GAP45, an essential component of the glideosome, has been shown to be palmitoylated in P. falciparum [26] and predicted to be palmitoylated in T. gondii $[30,81]$. This protein appears to be essential to maintain pellicle cohesion during the invasion process, thus membrane anchoring through lipidic posttranslational modifications should play a crucial role. In fact, it was reported that while N-terminal myristoylation and palmitoylation anchors TgGAP45 to the PM, palmitoylation on its C-terminal end allows its interaction with the IMC [81].

Polonais et al. [88] postulated that another T. gondii protein, myosin light chain 2 (MLC2), would have three palmitoylation sites in its N-terminal region, and that this region would be essential for membrane targeting. Furthermore, MLC2 interacts with variant D of myosin heavy chain (MyoD) which is also predicted to be palmitoylated, suggesting that such acylation would allow the interaction of proteins to form the complex MLC2-MyoD, which is part of the actin-myosin motor that accounts for the power necessary for parasite motility.

Tachyzoites treated with 2-bromopalmitate showed that their motility pattern was seriously altered [87]. Proteins that are part of the inner membrane complex (IMC) and the glideosome are predicted to be palmitoylated according to "in silico" analysis [30, 81, 88, 95], and this could explain the observed phenomena. It is also worth noting that in $T$. gondii parasites with reduced MIC2 expression-a putative palmitoylated protein [30]—exhibit less helical gliding [91]. Nowadays the question regarding the role of lipid modifications on these proteins still remains unsolved. Lipid modifications might be important for the correct assembly of multiprotein complexes or to allow synchronicity of the gliding, or perhaps they are involved in protein transport from their origin to their site of action.

\section{Protein Palmitoylation and Cell Shape}

Besides maintaining cell shape, the cytoskeleton provides essential support for vital functions such as cell motility and replication. In Toxoplasma, the glideosome is composed of myosin A, a myosin light chain 1 (MLC1) and glidingassociated proteins 40, 55, and 50 [96, 97]. As such, the IMC functions as a platform for the actin-myosin apparatus required for host-cell invasion and, together with subpellicular microtubules are important during replication as well. In addition, these subpellicular microtubules are also important for organelle replication, polarity, elongated cellshape, and for driving cell division [98, 99]. A complete review on cell-cycle, cytokinesis, and the structures that take part in them can be found elsewhere $[14,15,100]$. Cell division in apicomplexa involves DNA replication and chromosome segregation, nuclear division, and cytokinesis. The intracellular stages of apicomplexan parasites are quite diverse, and this diversity lies in the flexibility of the apicomplexan mode of replication. Whereas Toxoplasma divides through a mechanism termed endodyogeny, wherein two parasites are assembled within a mother cell giving $2,4,8$, and so forth tachyzoites per vacuole after several rounds of replication, Plasmodium and Sarcocystis dispense with cytokinesis and/or nuclear divisions for multiple cycles, forming a single multinucleate cell [15]. In Toxoplasma, daughter cells are encapsulated by the IMC and assembled by the cortical cytoskeleton (subpellicular microtubules and intermediate-filament like proteins) [101-106]. The apical polar ring microtubule-organizing center (MTOC) drives Toxoplasma budding [100], whereas the nuclear segregation is coordinated by the centrocone [107]. Therefore, both processes are separate events allowing disconnection of nuclear division and/from budding [100].

Long-term treatment of T. gondii intracellular tachyzoites with 2-bromopalmitate results in major changes in parasite morphology and shape [87]. Interestingly, this effect could only be observed in mature intracellular parasites but not in budding cells. Furthermore, treatment of either extracellular tachyzoites or tachyzoites that recently invaded cells did not show any change in their morphology. Consequently, palmitoylation seems to be important to maintain the morphology of the mature intracellular tachyzoite after the first round of replication, may be by palmitoylation of some cytoskeletal proteins. Thus, this posttranslational modification could be important to maintain cell shape. TgMORN1 is a protein associated to the cytoskeleton and to ring structures at the apical and posterior end of the IMC and to the centrocone [101]. This protein, which seems to play a role in maintaining the parasite's integrity during cytokinesis [102], is predicted to be palmitoylated. In 2010, Beck et al. [95] reported that three putative palmitoylated proteins of $T$. gondii's IMCIMC subcompartment proteins; TgISPs-were important for daughter cell formation. Furthermore, actin-like protein 1 (ALP1; toxodb accession TGME49_019280) is important for 
IMC protein delivery to the bud and is also predicted to be palmitoylated on cysteine residues 3 and 216. Two other proteins that are predicted to be palmitoylated and that may play a role in cytokinesis are striated fibre-assembling (toxodb accession TGME49_005670) and centrin 4 (toxodb accession TGME49_029910). In 2011, 14 new IMC proteins were found by data mining [104]. Interestingly these proteins showed differential expression during cytokinesis and most of them are predicted to be palmitoylated either at their $\mathrm{N}$ or C-terminus.

The possibility of having different PATs expressed at different regions of the mature IMC has been previously discussed [95]. Thus, it is also possible that these PATs could associate to the IMC at a later stage of parasite replication or only in mature tachyzoites, regulating the final step of parasite morphology.

\section{Conclusions and Future Trends}

Pathogenesis of diseases caused by apicomplexan parasites is highly dependent on two events: invasion to and replication within the host-cell. It is now clear that protein lipidation, and in particular palmitoylation, actively participates in the regulation of localization and function of proteins associated to mechanisms that are essential for the pathogenesis of the parasites.

To date, protein acylation in apicomplexans has been studied through genetic engineering methods such as mutagenesis or by in silico analysis, leaving aside more "classical" biochemical methods. The use of an inhibitor can provide evidence of the functional role of a modification in a given system. Thus, the evidence obtained when parasites were treated with 2-bromopalmitate representss the first evidence that protein palmitoylation in Apicomplexa affects parasite physiology [87]. For the next years, the efforts should focus on elucidating the palmitoyl proteome of apicomplexan parasites and the underlying mechanism of palmitoylation in apicomplexan pathogenesis. In this sense, in the recent years new approaches have been developed for characterizing protein palmitoylation. These approaches are more sensitive and faster than the traditional labeling, providing new opportunities for exploring this posttranslational modification. Bioorthogonal ligation reactions and azide-alkyne cycloadditions [108], palmitic acid analog 17octadecynoic acid (17-ODYA; [109]), and chemical reporters combined with orthogonal clickable fluorophores [110] are some of the new approaches that will shed light into the functional roles of lipidated proteins. The high sensitivity and efficiency of these approaches should assist the functional characterization of factors and drugs that modulate protein palmitoylation. The application of these biochemical methods together with proteomic tools and molecular biology will provide the means to resolve the palmitoyl-proteomes of model apicomplexans, as it has been carried out for trypanosomatids [111].

The enzymes involved in both the addition and removal of the palmitate residue are potential therapeutic targets for the treatment of parasitic diseases. Hence, the use of all the available tools will allow unraveling the molecular processes underlying palmitoylation in unicellular organisms and will shed light on the biology of these parasites.

\section{Acknowledgments}

This work was supported by ANPCyT Grant BID 1728 OCAR PICT 2010-1494 (MMC), National Council of Research (CONICET) PIP Grant 2011-0190 (MMC), and a National Institutes of Health-National Institute of Allergy and Infectious Diseases (NIH-NIAID) Grant AI083162 (MMC). M. M. Corvi is a researcher from the National Council of Research (CONICET) and UNSAM. A. M. Alonso and M. C. Caballero are Ph.D. fellows from CONICET.

\section{References}

[1] S. I. Hirst and L. A. Stapley, "Parasitology: the dawn of a new millennium," Parasitology Today, vol. 16, no. 1, pp. 1-3, 2000.

[2] C. Guinovart, M. M. Navia, M. Tanner, and P. L. Alonso, "Malaria: burden of disease," Current Molecular Medicine, vol. 6, no. 2, pp. 137-140, 2006.

[3] Y. Sukthana, "Toxoplasmosis: beyond animals to humans," Trends in Parasitology, vol. 22, no. 3, pp. 137-142, 2006.

[4] S. Tzipori and H. Ward, "Cryptosporidiosis: biology, pathogenesis and disease," Microbes and Infection, vol. 4, no. 10, pp. 1047-1058, 2002.

[5] J. G. Montoya and O. Liesenfeld, "Toxoplasmosis," The Lancet, vol. 363, no. 9425, pp. 1965-1976, 2004.

[6] G. I. McFadden, "The apicoplast," Protoplasma, vol. 248, pp. 641-650, 2010.

[7] F. Seeber and D. Soldati-Favre, "Metabolic pathways in the apicoplast of apicomplexa," International Review of Cell and Molecular Biology, vol. 281, pp. 161-228, 2010.

[8] S. A. Ralph, G. G. van Dooren, R. F. Waller et al., "Tropical infectious diseases: metabolic maps and functions of the Plasmodium falciparum apicoplast," Nature Reviews Microbiology, vol. 2, pp. 203-216, 2004.

[9] S. Besteiro, J. F. Dubremetz, and M. Lebrun, "The moving junction of apicomplexan parasites: a key structure for invasion," Cellular Microbiology, vol. 13, no. 6, pp. 797-805, 2011.

[10] J. S. Tyler, M. Treeck, and J. C. Boothroyd, "Focus on the ringleader: the role of AMA1 in apicomplexan invasion and replication," Trends in Parasitology, vol. 27, pp. 410-420, 2011.

[11] D. Bargieri, V. Lagal, I. Tardieux, and R. Ménard, "Host cell invasion by apicomplexans: what do we know?" Trends in Parasitology, vol. 28, no. 4, pp. 131-135, 2012.

[12] J. R. Radke, B. Striepen, M. N. Guerini, M. E. Jerome, D. S. Roos, and M. W. White, "Defining the cell cycle for the tachyzoite stage of Toxoplasma gondii," Molecular and Biochemical Parasitology, vol. 115, no. 2, pp. 165-175, 2001.

[13] S. Taylor, A. Barragan, C. Su et al., "A secreted serinethreonine kinase determines virulence in the eukaryotic pathogen Toxoplasma gondii," Science, vol. 314, no. 5806, pp. 1776-1780, 2006.

[14] M. J. Gubbels, M. White, and T. Szatanek, "The cell cycle and Toxoplasma gondii cell division: tightly knit or loosely stitched?" International Journal for Parasitology, vol. 38, no. 12, pp. 1343-1358, 2008. 
[15] B. Striepen, C. N. Jordan, S. Reiff, and G. G. van Dooren, "Building the perfect parasite: cell division in apicomplexa," PLoS Pathogens, vol. 3, no. 6, article e78, 2007.

[16] J. M. Berg, J. L. Tymoczko, and L. Stryer, "Covalent modification is a means of regulating enzyme activity," in Biochemistry, J. M. Berg, J. L. Tymoczko, and L. Stryer, Eds., W. H. Freeman, New York, NY, USA, 5th edition, 2002.

[17] R. S. Bhatnagar and J. I. Gordon, "Understanding covalent modifications of proteins by lipids: where cell biology and biophysics mingle," Trends in Cell Biology, vol. 7, no. 1, pp. 14-20, 1997.

[18] J. A. Porter, K. E. Young, and P. A. Beachy, "Cholesterol modification of hedgehog signaling proteins in animal development," Science, vol. 274, no. 5285, pp. 255-259, 1996.

[19] C. S. De Macedo, H. Shams-Eldin, T. K. Smith, R. T. Schwarz, and N. Azzouz, "Inhibitors of glycosyl-phosphatidylinositol anchor biosynthesis," Biochimie, vol. 85, no. 3-4, pp. 465-472, 2003.

[20] R. T. Eastman, F. S. Buckner, K. Yokoyama, M. H. Gelb, and W. C. Van Voorhis, "Fighting parasitic disease by blocking protein farnesylation," Journal of Lipid Research, vol. 47, no. 2, pp. 233-240, 2006.

[21] P. Grellier, D. Depoix, J. Schrével, and I. Florent, "Discovery of new targets for antimalarial chemotherapy," Parasite, vol. 15, no. 3, pp. 219-225, 2008.

[22] M. D. Resh, "Fatty acylation of proteins: new insights into membrane targeting of myristoylated and palmitoylated proteins," Biochimica et Biophysica Acta, vol. 1451, no. 1, pp. 1-16, 1999.

[23] G. L. Vilas, M. M. Corvi, G. J. Plummer, A. M. Seime, G. R. Lambkin, and L. G. Berthiaume, "Posttranslational myristoylation of caspase-activated p21-activated protein kinase 2 (PAK2) potentiates late apoptotic events," Proceedings of the National Academy of Sciences of the United States of America, vol. 103, no. 17, pp. 6542-6547, 2006.

[24] M. E. Linder and R. J. Deschenes, "New insights into the mechanisms of protein palmitoylation," Biochemistry, vol. 42, no. 15 , pp. 4317-4320, 2003.

[25] R. G. K. Donald and P. A. Liberator, "Molecular characterization of a coccidian parasite cGMP dependent protein kinase," Molecular and Biochemical Parasitology, vol. 120, no. 2, pp. 165-175, 2002.

[26] R. R. Rees-Channer, S. R. Martin, J. L. Green et al., "Dual acylation of the $45 \mathrm{kDa}$ gliding-associated protein (GAP45) in Plasmodium falciparum merozoites," Molecular and Biochemical Parasitology, vol. 149, no. 1, pp. 113-116, 2006.

[27] I. Russo, A. Oksman, and D. E. Goldberg, "Fatty acid acylation regulates trafficking of the unusual Plasmodium falciparum calpain to the nucleolus," Molecular Microbiology, vol. 72, no. 1, pp. 229-245, 2009.

[28] C. Kleuss and E. Krause, "G $\alpha$ s is palmitoylated at the Nterminal glycine," EMBO Journal, vol. 22, no. 4, pp. 826-832, 2003.

[29] R. B. Pepinsky, C. Zeng, D. Went et al., "Identification of a palmitic acid-modified form of human Sonic hedgehog," The Journal of Biological Chemistry, vol. 273, no. 22, pp. 1403714045, 1998.

[30] M. M. Corvi, L. G. Berthiaume, and M. G. De Napoli, "Protein palmitoylation in protozoan parasites," Frontiers in Bioscience, vol. 3, pp. 1067-1079, 2011.

[31] D. Michaelson, I. Ahearn, M. Bergo, S. Young, and M. Philips, "Membrane trafficking of heterotrimeric $\mathrm{G}$ proteins via the endoplasmic reticulum and golgi," Molecular Biology of the Cell, vol. 13, no. 9, pp. 3294-3302, 2002.

[32] S. Takida and P. B. Wedegaertner, "Heterotrimer formation, together with isoprenylation, is required for plasma membrane targeting of G $\beta \gamma$," The Journal of Biological Chemistry, vol. 278, no. 19, pp. 17284-17290, 2003.

[33] Y. I. Henis, J. F. Hancock, and I. A. Prior, "Ras acylation, compartmentalization and signaling nanoclusters (Review)," Molecular Membrane Biology, vol. 26, no. 1-2, pp. 80-92, 2009.

[34] D. A. Brown and E. London, "Functions of lipid rafts in biological membranes," Annual Review of Cell and Developmental Biology, vol. 14, pp. 111-136, 1998.

[35] M. Edidin, "The state of lipid rafts: from model membranes to cells," Annual Review of Biophysics \& Biomolecular Structure, vol. 32, pp. 257-283, 2003.

[36] L. Berthiaume, I. Deichaite, S. Peseckis, and M. D. Resh, "Regulation of enzymatic activity by active site fatty acylation. A new role for long chain fatty acid acylation of proteins," The Journal of Biological Chemistry, vol. 269, no. 9, pp. 6498-6505, 1994.

[37] M. M. Corvi, C. L. M. Soltys, and L. G. Berthiaume, "Regulation of mitochondrial carbamoyl-phosphate synthetase 1 activity by active site fatty acylation," The Journal of Biological Chemistry, vol. 276, no. 49, pp. 45704-45712, 2001.

[38] M. A. Kostiuk, B. O. Keller, and L. G. Berthiaume, "Palmitoylation of ketogenic enzyme HMGCS2 enhances its interaction with PPAR $\alpha$ and transcription at the Hmgcs2 PPRE," FASEB Journal, vol. 24, no. 6, pp. 1914-1924, 2010.

[39] O. A. Bizzozero and M. B. Lees, "Fatty acid acylation of rat brain myelin proteolipid protein in vitro: identification of the lipid donor," Journal of Neurochemistry, vol. 46, no. 2, pp. 630-636, 1986.

[40] G. James and E. N. Olson, "Fatty acylated proteins as components of intracellular signaling pathways," Biochemistry, vol. 29, no. 11, pp. 2623-2634, 1990.

[41] A. I. Magee, L. Gutierrez, I. A. McKay, C. J. Marshall, and A. Hall, "Dynamic fatty acylation of p21N-ras," EMBO Journal, vol. 6, no. 11, pp. 3353-3357, 1987.

[42] E. Ponimaskin and M. F. G. Schmidt, "Acylation of viral glycoproteins: structural requirements for palmitoylation of transmembrane proteins," Biochemical Society Transactions, vol. 23, no. 3, pp. 565-568, 1995.

[43] E. Ponimaskin and M. F. G. Schmidt, "Domain-structure of cytoplasmic border region is main determinant for palmitoylation of influenza virus hemagglutinin (H7)," Virology, vol. 249, no. 2, pp. 325-335, 1998.

[44] S. Shahinian and J. R. Silvius, "Doubly-lipid-modified protein sequence motifs exhibit long-lived anchorage to lipid bilayer membranes," Biochemistry, vol. 34, no. 11, pp. 38133822, 1995.

[45] I. Navarro-Lérida, A. Álvarez-Barrientos, F. Gavilanes, and I. Rodriguez-Crespo, "Distance-dependent cellular palmitoylation of de-novo-designed sequences and their translocation to plasma membrane subdomains," Journal of Cell Science, vol. 115, no. 15, pp. 3119-3130, 2002.

[46] L. A. Paige, M. J. S. Nadler, M. L. Harrison, J. M. Cassady, and R. L. Geahlen, "Reversible palmitoylation of the proteintyrosine kinase p56(lck)," The Journal of Biological Chemistry, vol. 268, no. 12, pp. 8669-8674, 1993.

[47] M. D. Resh, "Palmitoylation of ligands, receptors, and intracellular signaling molecules," Science's STKE, vol. 2006, no. 359, article re14, 2006. 
[48] J. Greaves, C. Salaun, Y. Fukata, M. Fukata, and L. H. Chamberlain, "Palmitoylation and membrane interactions of the neuroprotective chaperone cysteine-string protein," The Journal of Biological Chemistry, vol. 283, no. 36, pp. 25014 25026, 2008.

[49] A. E. El-Husseini, S. E. Craven, D. M. Chetkovich et al., "Dual palmitoylation of PSD-95 mediates its vesiculotubular sorting, postsynaptic targeting, and ion channel clustering," Journal of Cell Biology, vol. 148, no. 1, pp. 159-171, 2000.

[50] M. A. Kostiuk, M. M. Corvi, B. O. Keller et al., "Identification of palmitoylated mitochondrial proteins using a bioorthogonal azido-palmitate analogue," FASEB Journal, vol. 22, no. 3, pp. 721-732, 2008.

[51] S.-C. Chang and A. I. Magee, "Acyltransferases for secreted signalling proteins (Review)," Molecular Membrane Biology, vol. 26, no. 1-2, pp. 104-113, 2009.

[52] G. I. Miura and J. E. Treisman, "Lipid modification of secreted signaling proteins," Cell Cycle, vol. 5, no. 11, pp. 1184-1188, 2006.

[53] D. A. Mitchell, A. Vasudevan, M. E. Linder, and R. J. Deschenes, "Protein palmitoylation by a family of DHHC protein S-acyltransferases," Journal of Lipid Research, vol. 47, no. 6, pp. 1118-1127, 2006.

[54] Y. Ohno, A. Kihara, T. Sano, and Y. Igarashi, "Intracellular localization and tissue-specific distribution of human and yeast DHHC cysteine-rich domain-containing proteins," Biochimica et Biophysica Acta, vol. 1761, no. 4, pp. 474-483, 2006.

[55] J. Greaves, O. A. Gorleku, C. Salaun, and L. H. Chamberlain, "Palmitoylation of the SNAP25 protein family: specificity and regulation by DHHC palmitoyl transferases," The Journal of Biological Chemistry, vol. 285, no. 32, pp. 24629-24638, 2010.

[56] O. Rocks, M. Gerauer, N. Vartak et al., "The palmitoylation machinery is a spatially organizing system for peripheral membrane proteins," Cell, vol. 141, no. 3, pp. 458-471, 2010.

[57] T. L. Baker, H. Zheng, J. Walker, J. L. Coloff, and J. E. Buss, "Distinct rates of palmitate turnover on membrane-bound cellular and oncogenic H-Ras," The Journal of Biological Chemistry, vol. 278, no. 21, pp. 19292-19300, 2003.

[58] C. Huang, J. A. Duncan, A. G. Gilman, and S. M. Mumby, "Persistent membrane association of activated and depalmitoylated G protein $\alpha$ subunits," Proceedings of the National Academy of Sciences of the United States of America, vol. 96, no. 2, pp. 412-417, 1999.

[59] L. J. Robinson, L. Busconi, and T. Michel, "Agonistmodulated palmitoylation of endothelial nitric oxide synthase," The Journal of Biological Chemistry, vol. 270, no. 3, pp. 995-998, 1995.

[60] G. Siegel, G. Obernosterer, R. Fiore et al., "A functional screen implicates microRNA-138-dependent regulation of the depalmitoylation enzyme APT1 in dendritic spine morphogenesis," Nature Cell Biology, vol. 11, no. 6, pp. 705-716, 2009.

[61] M. Satou, Y. Nishi, J. Yoh, Y. Hattori, and H. Sugimoto, "Identification and characterization of acyl-protein thioesterase 1/lysophospholipase I as a ghrelin deacylation/lysophospholipid hydrolyzing enzyme in fetal bovine serum and conditioned medium," Endocrinology, vol. 151, no. 10, pp. 4765-4775, 2010.

[62] D. C. Yeh, J. A. Duncan, S. Yamashita, and T. Michel, "Depalmitoylation of endothelial nitric-oxide synthase by acyl-protein thioesterase 1 is potentiated by $\mathrm{Ca}^{2+}$ calmodulin," The Journal of Biological Chemistry, vol. 274, no. 46, pp. 33148-33154, 1999.

[63] W. Yang, D. Di Vizio, M. Kirchner, H. Steen, and M. R. Freeman, "Proteome scale characterization of human S-acylated proteins in lipid raft-enriched and non-raft membranes," Molecular and Cellular Proteomics, vol. 9, no. 1, pp. 54-70, 2010.

[64] A. A. Soyombo and S. L. Hofmann, "Molecular cloning and expression of palmitoyl-protein thioesterase 2 (PPT2), a homolog of lysosomal palmitoyl-protein thioesterase with a distinct substrate specificity," The Journal of Biological Chemistry, vol. 272, no. 43, pp. 27456-27463, 1997.

[65] L. A. Verkruyse and S. L. Hofmann, "Lysosomal targeting of palmitoyl-protein thioesterase," The Journal of Biological Chemistry, vol. 271, no. 26, pp. 15831-15836, 1996.

[66] L. A. Camp and S. L. Hofmann, "Purification and properties of a palmitoyl-protein thioesterase that cleaves palmitate from H-Ras," The Journal of Biological Chemistry, vol. 268, no. 30, pp. 22566-22574, 1993.

[67] M. E. Linder and R. J. Deschenes, "Palmitoylation: policing protein stability and traffic," Nature Reviews Molecular Cell Biology, vol. 8, no. 1, pp. 74-84, 2007.

[68] C. Salaun, J. Greaves, and L. H. Chamberlain, "The intracellular dynamic of protein palmitoylation," Journal of Cell Biology, vol. 191, no. 7, pp. 1229-1238, 2010.

[69] M. Veit and M. F. G. Schmidt, "Enzymatic depalmitoylation of viral glycoproteins with acyl-protein thioesterase 1 in vitro," Virology, vol. 288, no. 1, pp. 89-95, 2001.

[70] J. A. Follit, X. Li, Y. Vucica, and G. J. Pazour, "The cytoplasmic tail of fibrocystin contains a ciliary targeting sequence," Journal of Cell Biology, vol. 188, no. 1, pp. 21-28, 2010.

[71] S. Cevik, Y. Hori, O. I. Kaplan et al., "Joubert syndrome Arl13b functions at ciliary membranes and stabilizes protein transport in Caenorhabditis elegans," Journal of Cell Biology, vol. 188, no. 6, pp. 953-969, 2010.

[72] P. Oh and J. E. Schnitzer, "Segregation of heterotrimeric G proteins in cell surface microdomains: GQ binds caveolin to concentrate in caveolae, whereas gi and GS target lipid rafts by default," Molecular Biology of the Cell, vol. 12, no. 3, pp. 685-698, 2001.

[73] C. E. Ducker, L. K. Griffel, R. A. Smith et al., "Discovery and characterization of inhibitors of human palmitoyl acyltransferases," Molecular Cancer Therapeutics, vol. 5, no. 7, pp. 1647-1659, 2006.

[74] B. C. Jennings, M. J. Nadolski, Y. Ling et al., "2-bromopalmitate and 2-(2-hydroxy-5-nitro-benzylidene)-benzo[b] thiophen-3-one inhibit DHHC-mediated palmitoylation in vitro," Journal of Lipid Research, vol. 50, no. 2, pp. 233-242, 2009.

[75] J. T. Swarthout, S. Lobo, L. Farh et al., "DHHC9 and GCP16 constitute a human protein fatty acyltransferase with specificity for $\mathrm{H}$ - and N-Ras," The Journal of Biological Chemistry, vol. 280, no. 35, pp. 31141-31148, 2005.

[76] F. J. Dekker, O. Rocks, N. Vartak et al., "Small-molecule inhibition of APT1 affects Ras localization and signaling," Nature Chemical Biology, vol. 6, no. 6, pp. 449-456, 2010.

[77] L. D. Sibley, "Invasion and intracellular survival by protozoan parasites," Immunological Reviews, vol. 240, no. 1, pp. 72-91, 2011.

[78] M. K. Shaw, "Cell invasion by Theileria sporozoites," Trends in Parasitology, vol. 19, no. 1, pp. 2-6, 2003. 
[79] A. Keeley and D. Soldati, "The glideosome: a molecular machine powering motility and host-cell invasion by Apicomplexa," Trends in Cell Biology, vol. 14, no. 10, pp. 528532, 2004.

[80] V. Carruthers and J. C. Boothroyd, "Pulling together: an integrated model of Toxoplasma cell invasion," Current Opinion in Microbiology, vol. 10, no. 1, pp. 83-89, 2007.

[81] K. Frénal, V. Polonais, J. B. Marq, R. Stratmann, J. Limenitakis, and D. Soldati-Favre, "Functional dissection of the apicomplexan glideosome molecular architecture," Cell Host and Microbe, vol. 8, no. 4, pp. 343-357, 2010.

[82] V. B. Carruthers and L. D. Sibley, "Sequential protein secretion front three distinct organelles of Toxoplasma gondii accompanies invasion of human fibroblasts," European Journal of Cell Biology, vol. 73, no. 2, pp. 114-123, 1997.

[83] D. G. Mordue, S. Håkansson, I. Niesman, and L. David Sibley, "Toxoplasma gondii resides in a vacuole that avoids fusion with host cell endocytic and exocytic vesicular trafficking pathways," Experimental Parasitology, vol. 92, no. 2, pp. 8799, 1999.

[84] M. Asada, Y. Goto, K. Yahata et al., "Gliding motility of babesia bovis merozoites visualized by time-lapse video microscopy," PLoS ONE, vol. 7, no. 4, article e35227, 2012.

[85] A. M. Gurnett, P. A. Liberator, P. M. Dulski et al., "Purification and molecular characterization of cGMPdependent protein kinase from Apicomplexan parasites. A novel chemotherapeutic target," The Journal of Biological Chemistry, vol. 277, no. 18, pp. 15913-15922, 2002.

[86] B. Nare, J. J. Allocco, P. A. Liberator, and R. G. K. Donald, "Evaluation of a cyclic GMP-dependent protein kinase inhibitor in treatment of murine toxoplasmosis: gamma interferon is required for efficacy," Antimicrobial Agents and Chemotherapy, vol. 46, no. 2, pp. 300-307, 2002.

[87] A. M. Alonso, V. M. Coceres, M. G. De Napoli, A. F. Nieto Guil, S. O. Angel, and M. M. Corvi, "Protein palmitoylation inhibition by 2-bromopalmitate alters gliding, host cell invasion and parasite morphology in Toxoplasma gondii," Molecular and Biochemical Parasitology, vol. 184, no. 1, pp. 39-43, 2012.

[88] V. Polonais, B. Javier Foth, K. Chinthalapudi et al., "Unusual anchor of a motor complex (MyoD-MLC2) to the plasma membrane of Toxoplasma gondii," Traffic, vol. 12, no. 3, pp. 287-300, 2011.

[89] M. D. Resh, "Use of analogs and inhibitors to study the functional significance of protein palmitoylation," Methods, vol. 40, no. 2, pp. 191-197, 2006.

[90] R. A. Coleman, P. Rao, R. J. Fogelsong, and E. S. G. Bardes, "2-Bromopalmitoyl-CoA and 2-bromopalmitate: promiscuous inhibitors of membrane-bound enzymes," Biochimica et Biophysica Acta, vol. 1125, no. 2, pp. 203-209, 1992.

[91] M. H. Huynh and V. B. Carruthers, "Toxoplasma MIC2 is a major determinant of invasion and virulence," PLoS Pathogens, vol. 2, no. 8, p. e84, 2006.

[92] D. P. Blake, K. J. Billington, S. L. Copestake et al., "Genetic mapping identifies novel highly protective antigens for an apicomplexan parasite," PLoS Pathogens, vol. 7, no. 2, Article ID e1001279, 2011.

[93] X. Cui, T. Lei, D. Yang, P. Hao, B. Li, and Q. Liu, “Toxoplasma gondii immune mapped protein-1 (TgIMP1) is a novel vaccine candidate against toxoplasmosis," Vaccine, vol. 30, no. 13, pp. 2282-2287, 2012.

[94] X. Cui, T. Lei, D. Y. Yang, P. Hao, and Q. Liu, "Identification and characterization of a novel Neospora caninum immune mapped protein 1," Parasitology, vol. 139, no. 8, pp. 9981004, 2012.

[95] J. R. Beck, I. A. Rodriguez-Fernandez, J. C. de Leon et al., "A novel family of Toxoplasma IMC proteins displays a hierarchical organization and functions in coordinating parasite division," PLoS Pathogens, vol. 6, no. 9, Article ID e01094, 2010.

[96] D. Soldati and M. Meissner, "Toxoplasma as a novel system for motility," Current Opinion in Cell Biology, vol. 16, no. 1, pp. 32-40, 2004.

[97] T. M. Johnson, Z. Rajfur, K. Jacobson, and C. J. Beckers, "Immobilization of the type XIV myosin complex in Toxoplasma gondii," Molecular Biology of the Cell, vol. 18, no. 8, pp. 3039-3046, 2007.

[98] N. S. Morrissette and L. D. Sibley, "Disruption of microtubules uncouples budding and nuclear division in Toxoplasma gondii," Journal of Cell Science, vol. 115, no. 5, pp. 1017-1025, 2002.

[99] B. A. Nichols and M. L. Chiappino, "Cytoskeleton of Toxoplasma gondii," Journal of Protozoology, vol. 34, no. 2, pp. 217-226, 1987.

[100] N. S. Morrissette and L. D. Sibley, "Cytoskeleton of apicomplexan parasites," Microbiology and Molecular Biology Reviews, vol. 66, no. 1, pp. 21-38, 2002.

[101] M. J. Gubbels, S. Vaishnava, N. Boot, J. F. Dubremetz, and B. Striepen, "A MORN-repeat protein is a dynamic component of the Toxoplasma gondii cell division apparatus," Journal of Cell Science, vol. 119, no. 11, pp. 2236-2245, 2006.

[102] A. T. Heaslip, F. Dzierszinski, B. Stein, and K. Hu, "TgMORN1 is a key organizer for the basal complex of Toxoplasma gondii," PLoS Pathogens, vol. 6, no. 2, Article ID e1000754, 2010.

[103] A. Lorestani, L. Sheiner, K. Yang et al., "A Toxoplasma MORN1 null mutant undergoes repeated divisions but is defective in basal assembly, apicoplast division and cytokinesis," PLoS ONE, vol. 5, no. 8, Article ID e12302, 2010.

[104] B. R. Anderson-White, F. D. Ivey, K. Cheng et al., "A family of intermediate filament-like proteins is sequentially assembled into the cytoskeleton of Toxoplasma gondii," Cellular Microbiology, vol. 13, no. 1, pp. 18-31, 2011.

[105] K. Hu, J. Johnson, L. Florens et al., "Cytoskeletal components of an invasion machine-the apical complex of Toxoplasma gondii," PLoS Pathogens, vol. 2, no. 2, pp. 0121-0138, 2006.

[106] K. Hu, "Organizational changes of the daughter basal complex during the parasite replication of Toxoplasma gondii," PLoS Pathogens, vol. 4, no. 1, p. e10, 2008.

[107] C. F. Brooks, M. E. Francia, M. Gissot, M. M. Croken, K. Kim, and B. Striepen, "Toxoplasma gondii sequesters centromeres to a specific nuclear region throughout the cell cycle," Proceedings of the National Academy of Sciences of the United States of America, vol. 108, no. 9, pp. 3767-3772, 2011.

[108] H. C. Hang, J. P. Wilson, and G. Charron, "Bioorthogonal chemical reporters for analyzing protein lipidation and lipid trafficking," Accounts of Chemical Research, vol. 44, no. 9, pp. 699-708, 2011.

[109] B. R. Martin, C. Wang, A. Adibekian, S. E. Tully, and B. F. Cravatt, "Global profiling of dynamic protein palmitoylation," Nature Methods, vol. 9, no. 1, pp. 84-89, 2012.

[110] M. M. Zhang, L. K. Tsou, G. Charron, A. S. Raghavan, and H. C. Hang, "Tandem fluorescence imaging of dynamic Sacylation and protein turnover," Proceedings of the National Academy of Sciences of the United States of America, vol. 107, no. 19, pp. 8627-8632, 2010. 
[111] B. T. Emmer, E. S. Nakayasu, C. Souther et al., "Global analysis of protein palmitoylation in African trypanosomes," Eukaryotic Cell, vol. 10, no. 3, pp. 455-463, 2011. 

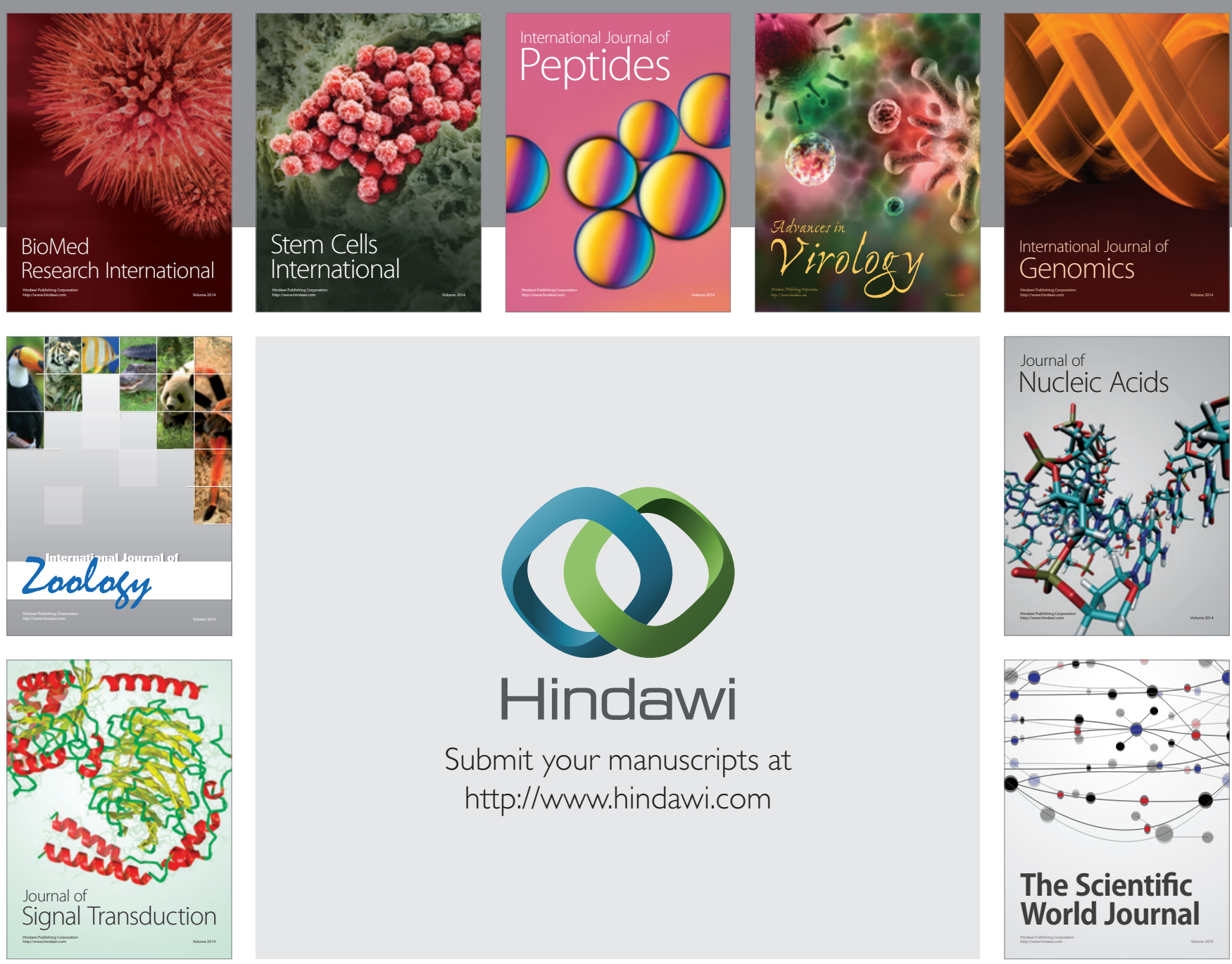

Submit your manuscripts at

http://www.hindawi.com
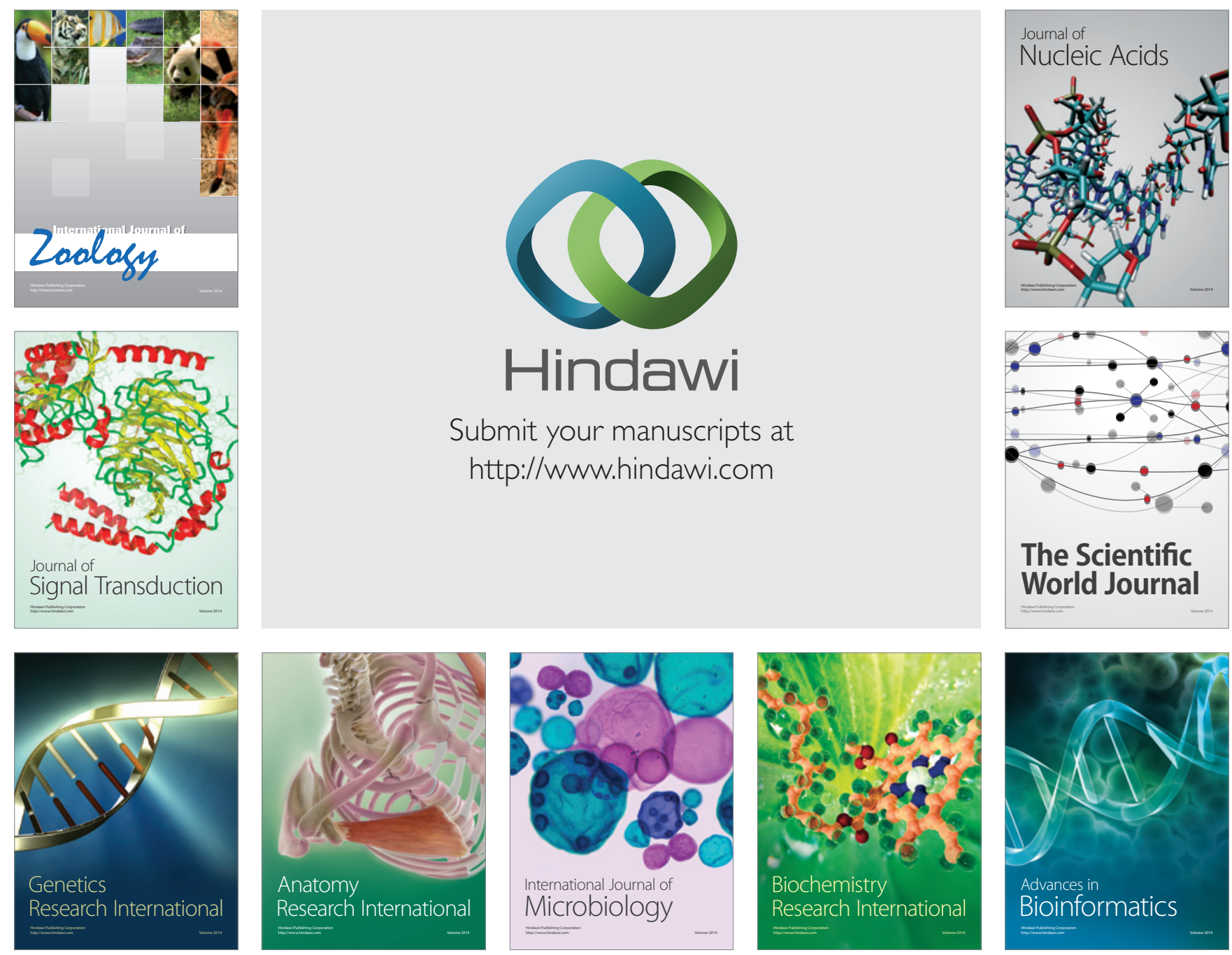

The Scientific World Journal
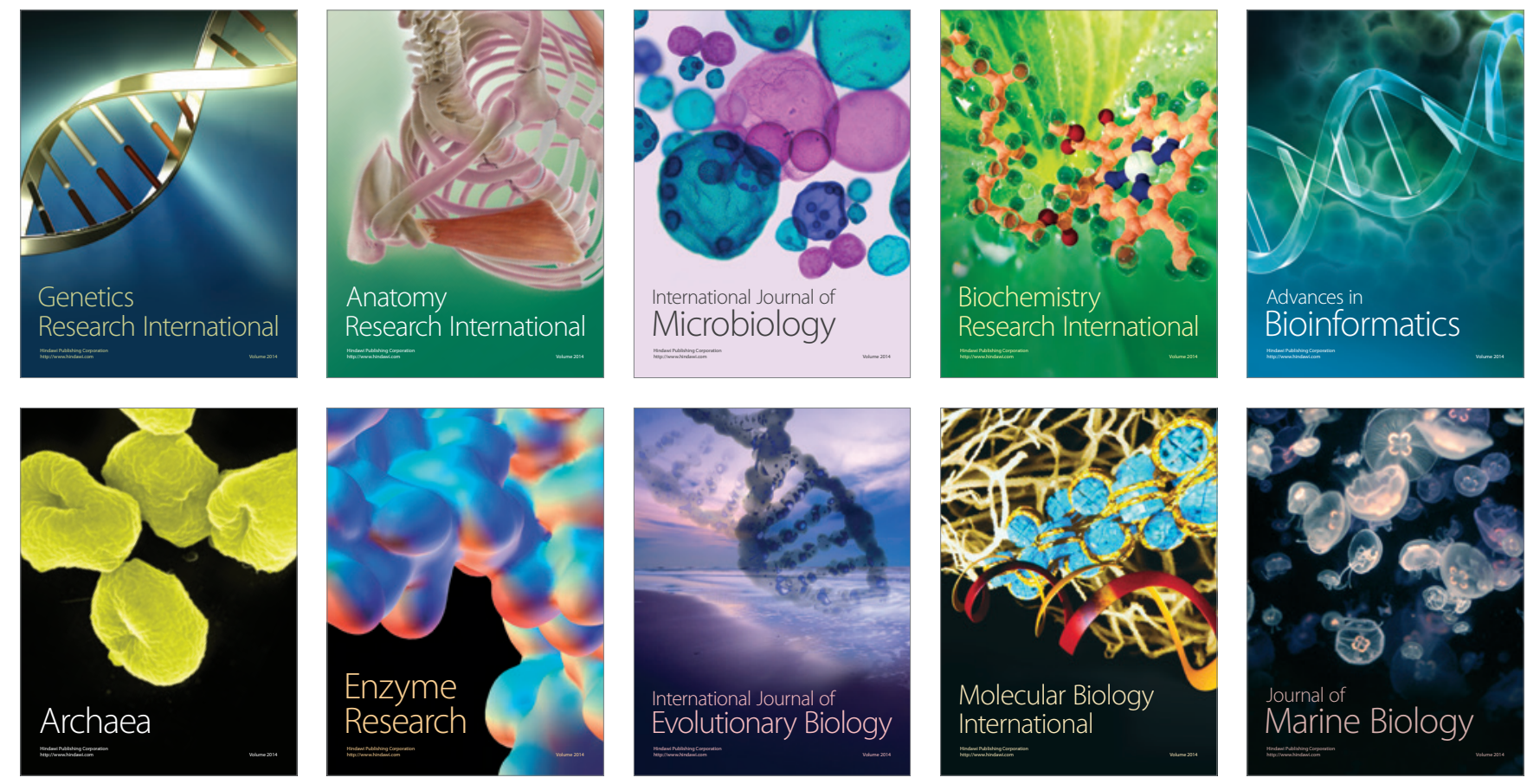